\title{
Introduction to Pharmaceutical Calculations - Fourth edition Judith A. Rees, Ian Smith and Jennie Watson
}

Pharmaceutical Press, London, UK., 2015

This book by Rees, Smith and Watson is applicable to pharmacy students, pharmacy technician students, as well as instructors. The book is divided into 13 chapters and covers topics such as concentrations, dilutions and dosage calculation, that most pharmacy practitioners encounter. The authors have primarily used the concept of proportions to approach most of the mathematical problems covered in this book. The use of a consistent approach allows for continuity from one chapter to the next, and teaches students a single approach that enables solving most pharmacy-related mathematical problems. The drawback, however, is that this one single approach may not suit all readers and learning styles.

The text is well laid out and easy to follow. Concepts are discussed thoroughly while some areas, e.g., density, specific gravity and significant figures, can benefit from more in depth discussions. The practice questions are relevant to most situations and are well-used to illustrate the concepts of interest. The number of examples and practice questions are sufficient. It is evident that the book is primarily intended for students in the UK since the British Pharmacopeia and British National Formulary are its main references. While readers outside of the UK will not have difficulty understanding the concepts, they will find some of the references not applicable. The authors included some discussion on infusion rates and concentrations. However, the book can benefit from inclusion of more in depth discussions and examples relevant to hospital practice (e.g., concentrations and the mixing of intravenous electrolyte solutions). The inclusion of access to online videos is a welcomed addition and can appeal to students with various learning styles.

In conclusion, this is a useful text for students and instructors. The practice questions in this book are relevant to daily practice and can be used as a source of additional practice for students.

Corresponding Author: Wilson Cheng, BSc. Pharm., PhD. Faculty of Pharmacy and Pharmaceutical Sciences; University of Alberta Hospital/Stollery Children Hospital, Alberta Health Services, Edmonton, Alberta, Canada, E-mail: wkcheng@ualberta.ca 